\title{
Alteración del campo visual no siempre es glaucoma. Reporte de caso
}

\section{Visual field changes are not always glaucoma. Case report}

\author{
Gabriel E. Ortiz, Lina M. Pineda* y Laura C. Vargas \\ Facultad de Medicina, Universidad Nacional de Colombia, Bogotá, Colombia
}

\section{Resumen}

Introducción: Las lesiones de estructuras en vecindad al quiasma y los nervios ópticos en su porción intracraneana pueden alterar la vía visual y generar algunas pérdidas del campo visual. Resumen del caso: Se presenta un reporte de caso de una lesión vascular en el sifón carotídeo que fue encontrada y estudiada por una alteración en el campo visual dentro del estudio por sospecha de glaucoma. Conclusiones: La evaluación integral y detallada permite hacer un diagnóstico correcto, evitando errores que pueden generar alta morbilidad y mortalidad.

Palabras clave: Glaucoma. Campo visual. Arteria carótida interna. Sifón carotídeo. Aneurisma.

\begin{abstract}
Introduction: Pathologic lesions in every structure surrounding optic chiasm and intracranial optic nerves have the potential to harm the visual pathway and cause a possible disturbance in the visual field. Case summary: We report a case of a carotid aneurism discovered and studied in the context of an anomaly in the visual field in a patient being studied as a glaucoma suspect. Conclusions: An integral and detailed evaluation guides to a certain diagnosis, avoiding errors that might cause high morbidity and mortality.
\end{abstract}

Key words: Glaucoma. Visual field. Internal carotid artery. Carotid syphon. Aneurysm.

\section{Introducción}

Los campos visuales son una de las herramientas fundamentales en el diagnóstico y el seguimiento de los pacientes con glaucoma o sospecha de glaucoma; sin embargo, hay numerosas patologías oftalmológicas y neurológicas que pueden originar defectos campimétricos similares a los del glaucoma o que pueden coincidir con la neuropatía glaucomatosa, por lo que debe tenerse especial cuidado en el análisis de los hallazgos clínicos y paraclínicos para no caer en la tendencia a atribuir todas las ateraciones exclusivamente al glaucoma.

Algunas de las patologías no glaucomatosas que pueden afectar el campo visual son enfermedades del nervio óptico, lesiones del quiasma óptico, lesiones neurológicas retroquiasmáticas, patología macular y retiniana, ptosis y peudoptosis por dermatochalasis marcada, defectos refractivos mal corregidos, artefactos e inexperiencia del paciente en la realización de la prueba. Las lesiones neurológicas que hay que tener
Correspondencia:

*Lina M. Pineda

E-mail: linamarcepineda@gmail.com DOI: 10.24875/RSCO.M2100000

CC BY-NC-ND (http://creativecommons.org/licenses/by-nc-nd/4.0/) 
cuenta son tumores, infecciones y enfermedades vasculares, como isquemia o aneurismas ${ }^{1}$.

Los aneurismas intracraneales ocurren en cerca del $5 \%$ de la población general ${ }^{2}$ y pueden comprometer los campos visuales o la agudeza visual, en especial los de la arteria carótida interna $(\mathrm{ACl})$, que tiene una relación anatómica estrecha con el nervio óptico y el quiasma ${ }^{3,4}$, principalmente en sus porciones cavernosa, clinoidea y oftálmica. El sifón carotídeo es una parte de la $\mathrm{ACl}$ en forma de $\mathrm{S}$ que comienza en la curva posterior de la porción cavernosa y termina en la bifurcación en arteria comunicante posterior y arteria cerebral media $^{5}$.

En el presente artículo se presenta el caso de una paciente a quien se le diagnosticó un aneurisma del sifón carotídeo izquierdo durante el estudio por sospecha de glaucoma. Es importante porque ejemplifica cómo una patología potencialmente mortal puede confundirse con un glaucoma por las alteraciones en el campo visual.

\section{Presentación del caso}

Mujer de 66 años a quien se había diagnosticado glaucoma en otra institución 15 días antes y se inició tratamiento tópico con latanoprost y maleato de timolol. Fue remitida por presentar ángulos estrechos para evaluar la necesidad de iridotomía láser. Tenía antecedente de hipertensión arterial sistémica y diabetes mellitus diagnosticada 2 años antes. Los antecedentes familiares eran negativos para glaucoma. Al examen oftalmológico se halló una agudeza visual sin corrección de 20/20 en el ojo derecho (OD) y 20/30 en el ojo izquierdo (OI), cámara anterior grado I en ambos ojos, la gonioscopía evidenció ángulos $<15^{\circ}$ en todos los cuadrantes con pigmento 1+/2++, presión intraocular $10 / 11 \mathrm{mmHg}$ (con medicación), el examen del fondo mostró nervio óptico rosado, relación copa-disco 0.3 en ambos ojos $y$ alteraciones en la retina nasal e inferior en el $\mathrm{Ol}$ (Figs. 1 y 2). La ultrabiomicroscopía reportó ángulos $<10^{\circ}$, paquimetría óptica central en OD $533 \mu \mathrm{m}$ y OI $542 \mu \mathrm{m}$, campo visual central Sita-Standard 24-2 reportado con defecto leve en el OD y con defecto grave en el OI, compatible con daño por glaucoma (Fig. 3).

Con estos hallazgos se replanteó el diagnóstico, pues aunque la paciente tenía ángulos ocluibles y alteraciones del campo visual compatibles con glaucoma, los hallazgos del nervio óptico no se correlacionaban bien con las alteraciones campimétricas. Al revisar los campos visuales se evidenció una tendencia a respetar el meridiano vertical en localizaciones temporales en ambos ojos, más marcada en el OI, por lo que se

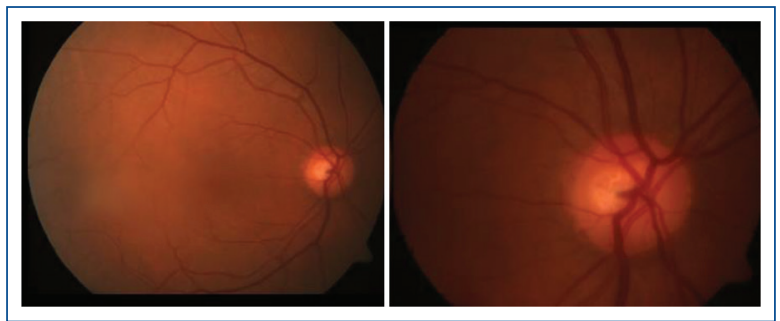

Figura 1. Fotos del polo posterior del ojo derecho. Se observa retina aplicada, arcadas vasculares normales, relación E/D 0.3, mácula sin brillo y sin cambios del epitelio pigmentario de la retina.

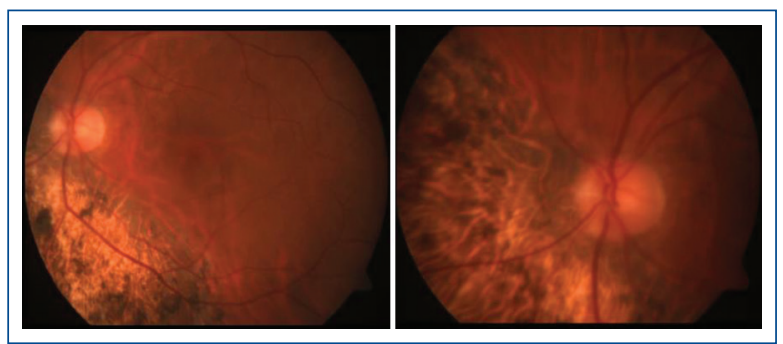

Figura 2. Fotos del polo posterior del ojo izquierdo. Se observa retina aplicada, coroidosis con zonas de hipopigmentación e hiperpigmentación en cuadrantes nasales, arcadas vasculares normales, relación E/D 0.2, mácula sin brillo.

decidió descartar una lesión quiasmática. Se solicitó tomografía de coherencia óptica (OCT) de nervios ópticos y tomografía computarizada (TC) cerebral simple y contrastada, se suspendió la medicación hipotensora y se practicó iridotomía láser en ambos ojos.

Tres meses después, la OCT Cirrus 2000 de nervios ópticos de noviembre de 2015 reportó una capa de fibras nerviosas dentro de los límites normales, igual que los mapas de espesores y desviación (Fig. 4); hallazgos sin evidencia de lesión glaucomatosa en ambos ojos. La TC cerebral evidenció una lesión aneurismática en el sifón carotídeo izquierdo (Figs. 5 y 6), por lo que se remitió a neurología de forma prioritaria.

La paciente regresó un año después a consulta, después de recibir tratamiento de embolización con coil endovascular del aneurisma por parte de radiología intervencionista (Fig. 7), procedimiento que fue realizado sin complicaciones.

\section{Discusión}

Los aneurismas intracraneales ocurren en cerca del $5 \%$ de la población general ${ }^{2}$, siendo la mayoría asintomáticos 


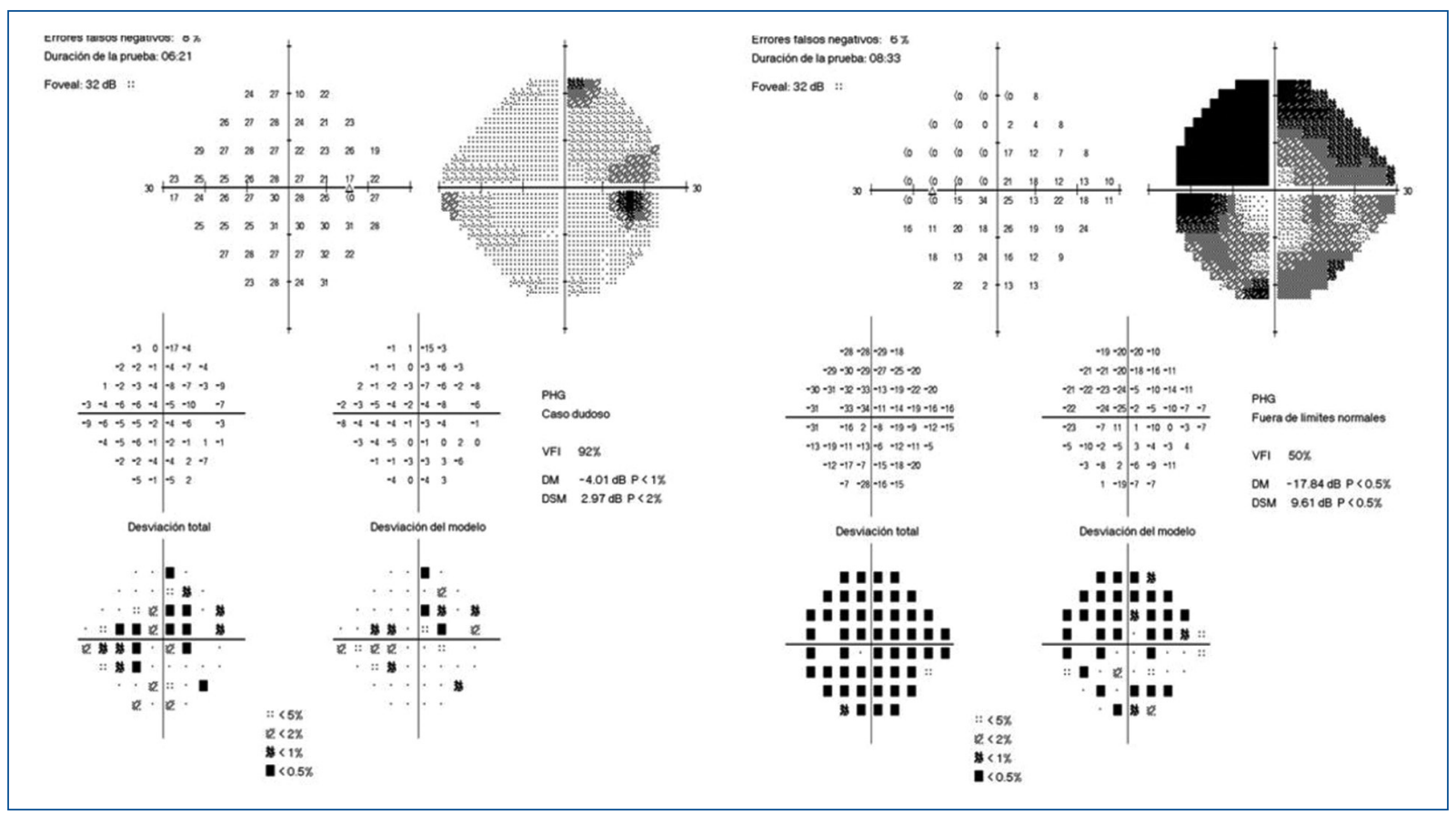

Figura 3. Campo visual computarizado. Se observan defectos en ambos ojos que tienen tendencia a respetar el meridiano vertical.

antes de su rotura 6 . No obstante, dependiendo de su localización, pueden generar síntomas oftalmológicos, como disminución de la agudeza visual, alteraciones del campo visual y oftalmoplejía. El diagnóstico no es fácil dado que no existe una presentación típica y puede ser confundido con glaucoma de forma frecuente ${ }^{6}$. Los aneurismas que más causan síntomas oftalmológicos son los de la $\mathrm{ACl}$, la arteria oftálmica y la arteria comunicante anterior ${ }^{5}$.

La clasificación anatómica de la $\mathrm{ACl}$ empleada actualmente la define en siete segmentos según la anatomía circundante y los compartimentos a través de los cuales viaja, enumerados en la dirección del flujo sanguíneo anterógrado: cervical (C1), petroso (C2), rasgado (C3), cavernoso (C4), clinoideo (C5), oftálmico (C6) y comunicante (C7). El asa anterior, también conocida como sifón carotídeo, contiene el segmento cavernoso distal, el segmento clinoideo y el segmento oftálmico, así como los anillos durales proximales y distales, encontrándose justamente por debajo del quiasma óptico ${ }^{5,7}$. El sifón carotídeo fue descrito por primera vez por Moniz en 1927, haciendo referencia a la apariencia que tiene la $\mathrm{ACl}$ en su porción intracraneana al visualizarla en la arteriografía ${ }^{8}$.
Los aneurismas intracreaneales con frecuencia ocurren en la $\mathrm{ACl}^{9-11}$. Un estudio que evaluó las características de los aneurismas intracraneales en un periodo de 9 años halló que el $11 \%$ de aproximadamente 700 pacientes tenían un aneurisma de la porción oftálmica de la $\mathrm{ACl}^{12}$, y otros autores describen un $8.3 \%$ en 604 pacientes ${ }^{13}$. En ambos estudios fue más frecuente en mujeres en la quinta a séptima décadas de la vida, dato que es congruente con los resultados de otros estudios $^{14}$. La mayoría de los aneurismas se mantienen asintomáticos hasta su rotura, pero un 10\% pueden presentar síntomas. Cuando hay un aneurisma que comprime el nervio óptico o el quiasma puede disminuir la visión, ya sea de forma insidiosa o aguda ${ }^{3}$. Los aneurismas de la $\mathrm{ACl}$ pueden generar diferentes alteraciones en el campo visual, dependiendo del nivel de compresión. La lesión clásica es el escotoma de la unión cuando hay una compresión en la porción final del nervio óptico, donde se une con el quiasma; a este nivel, las fibras del campo temporal del ojo contralateral ya se han decusado. En la campimetría se observa entonces una lesión central o difusa en el ojo homolateral a la lesión, y en el ojo contralateral se aprecia una cuadrantopsia del campo temporal superior ${ }^{3,15}$. Sin embargo, la alteración del campo visual puede variar 


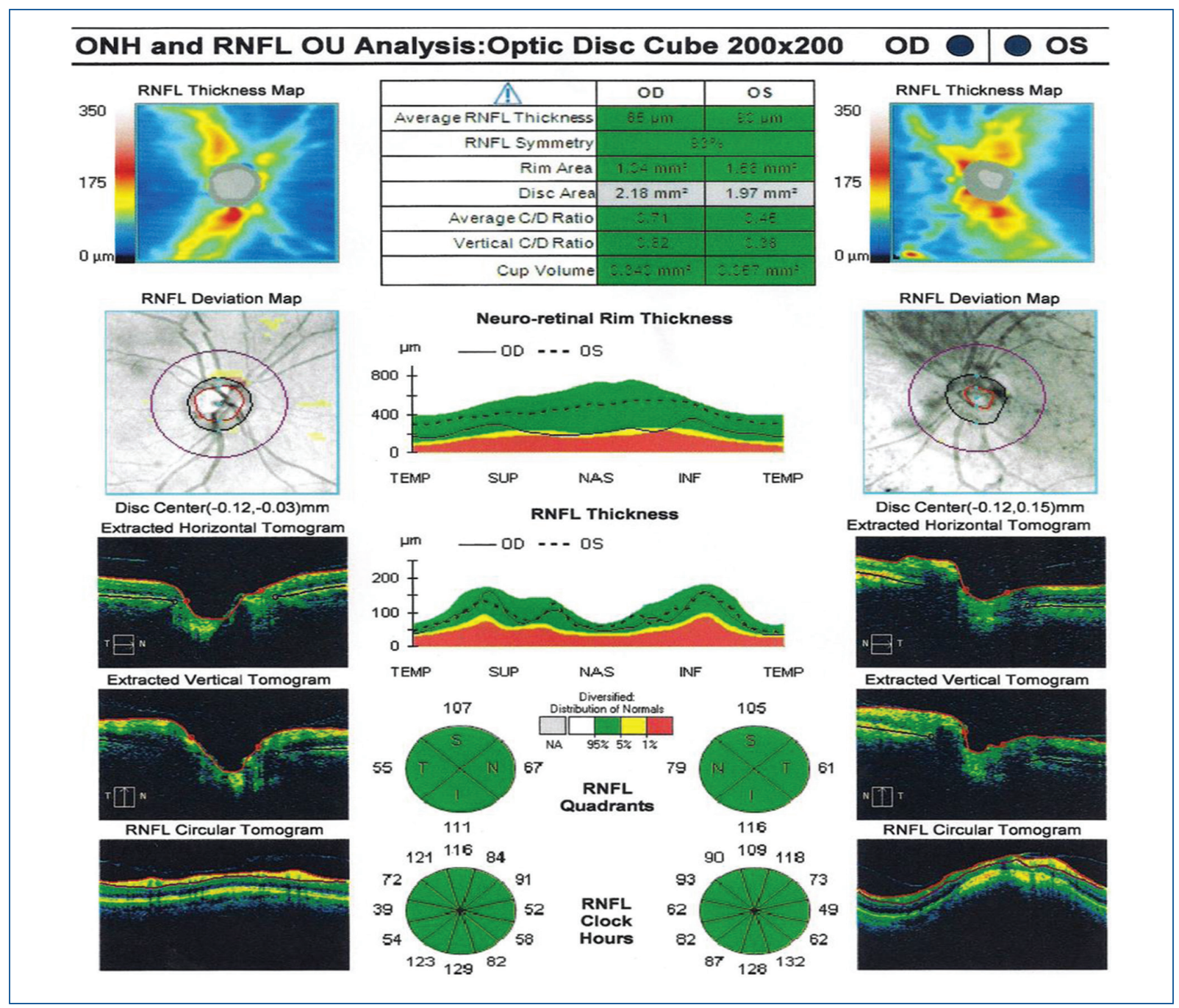

Figura 4. Tomografía de coherencia óptica Cirrus 2000.
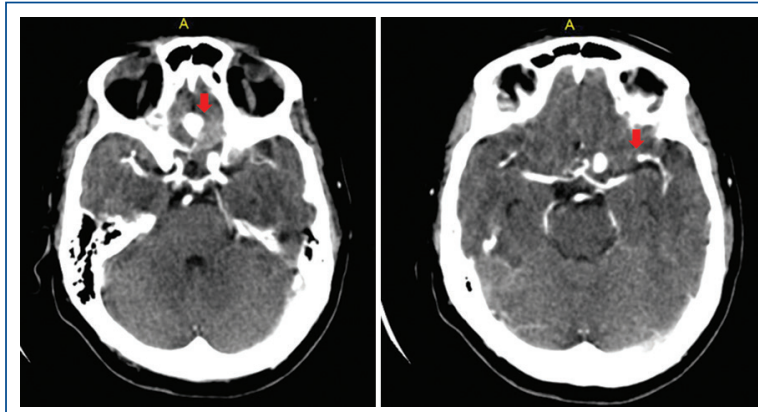

Figura 5. Tomografía computarizada de cráneo con contraste. Corte axial en el cual se observa una lesión aneurismática del sifón carotídeo izquierdo.

enormemente, no solo por la localización del aneurisma, sino también por las variaciones anatómicas que existen de un individuo a otro ${ }^{16}$. En los estudios antes mencionados hubo síntomas oftalmológicos en el $36 \%$ y el $50 \%$ de los pacientes, respectivamente, siendo la alteración del campo visual y la disminución de la visión los síntomas más frecuentes. En ambos estudios fueron más frecuentes las lesiones campimétricas unilaterales, con predominio del campo nasal inferior, con gran variabilidad en la presentación, desde defectos altitudinales hasta defectos totales. Cabe anotar que en ninguno de los dos estudios se halló escotoma de la unión.

En el caso clínico expuesto, el estudio por sospecha de glaucoma comenzó por mostrar ángulos estrechos, «confirmando» el diagnóstico de glaucoma con los hallazgos en el campo visual, aunque los nervios ópticos tenían una apariencia sana y las presiones intraoculares habían sido normales en tomas aisladas. El 


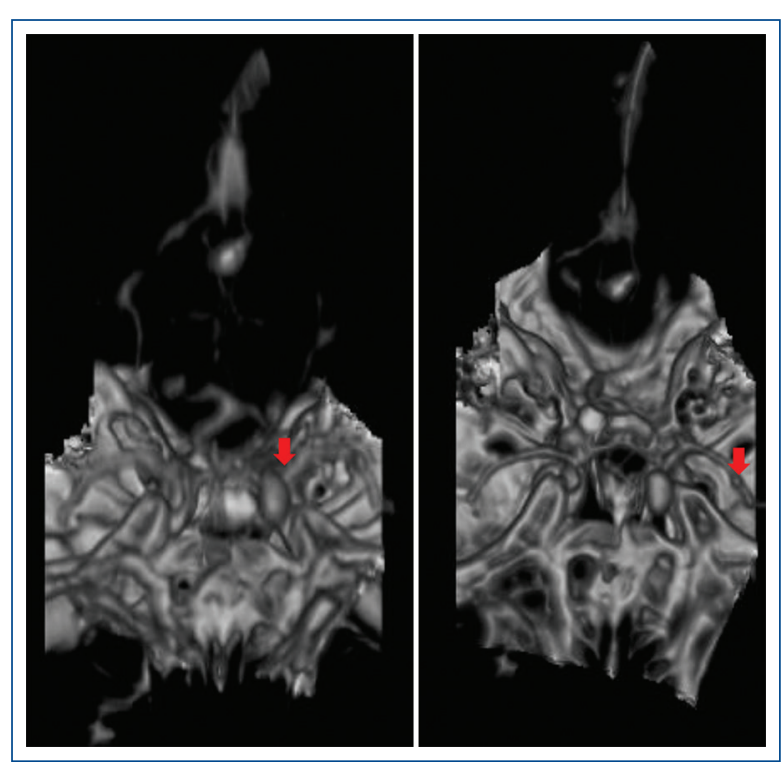

Figura 6. Tomografía. En la reconstrucción 3D se observa una lesión aneurismática del sifón carotídeo izquierdo en su porción oftálmica.

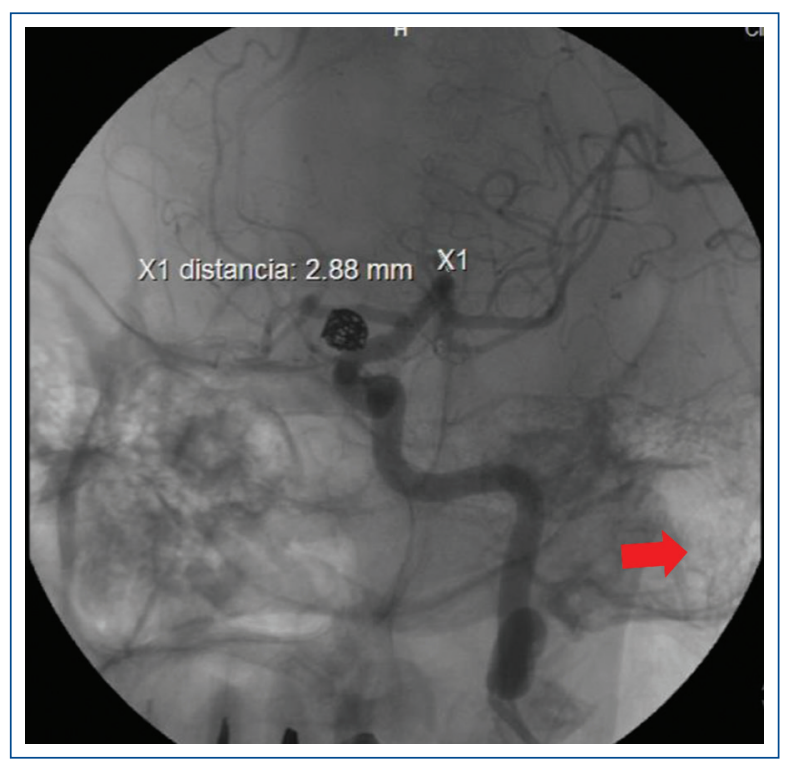

Figura 7. Arteriografía después de realizado el tratamiento endovascular. Se observa el coil ubicado en el área del aneurisma.

diagnóstico de glaucoma se sustentó primordialmente en los defectos del campo visual, de interpretación desafiante, pues la paciente presentaba además una zona de coroidosis importante que podía explicar alguna alteración del campo visual del OI. No obstante, la evaluación cuidadosa de los campos visuales con el hallazgo de la tendencia a respetar el meridiano vertical permitió sospechar una lesión neurológica y adelantar los estudios que condujeron al diagnóstico y el tratamiento oportunos de una lesión vascular potencialmente incapacitante o mortal.

\section{Recomendaciones}

Al evaluar pacientes con diagnósticos realizados en otras instituciones, la mayoría bien estudiados y manejados, debemos enfocarlos siempre como casos nuevos, no dar por ciertos los diagnósticos previos y analizar todos los hallazgos en forma cuidadosa, teniendo en mente los diagnósticos diferenciales, incluso los menos frecuentes, ya que en ocasiones podemos detectar otras afecciones incluso más graves 0 , como en este caso, potencialmente letales. Esto es válido también para pacientes con diagnósticos hechos por uno mismo; ante hallazgos nuevos o cuando no haya correlación entre los diferentes hallazgos, es importante hacer una reevaluación del caso. En cuanto al diagnóstico de glaucoma, debemos recordar la importancia de tener en cuenta todos los factores antes de etiquetar a un paciente como glaucomatoso e iniciar el tratamiento, dadas sus implicaciones en todos los aspectos de la vida del paciente y del sistema de salud.

\section{Conflicto de intereses}

Los autores declaran que no tienen conflictos de intereses.

\section{Financiamiento}

La presente investigación no ha recibido ninguna beca específica de agencias de los sectores públicos, comercial, o sin ánimo de lucro.

\section{Responsabilidades éticas}

Protección de personas y animales. Los autores declaran que para esta investigación no se han realizado experimentos en seres humanos ni en animales.

Confidencialidad de los datos. Los autores declaran que han seguido los protocolos de su centro de trabajo sobre la publicación de datos de pacientes.

Derecho a la privacidad y consentimiento informado. Los autores han obtenido el consentimiento 
informado de los pacientes y/o sujetos referidos en el artículo.

\section{Bibliografía}

1. Heijl A, Patella VM. Essential perimetry, the field analyzer primer. 3rd ed. California: Carl Zeiss Meditec; 2002.

2. García Carreira MC, Cánovas Vergé D, Marco Igual M, Hervàs Pujol M. Aneurismas supraclinoideos bilaterales asociados de déficit visual progresivo. Neurología. 2013;28:119-29.

3. Lanning B. Kline. Optic Nerve Disorders. En: Thomas J. Walsh. Visual Fields. Third Edition. New York: Oxford University Press, Inc.; 2011.

4. Bhat DI, Sampath S. Anterior communicating artery aneurysm presenting as monocular blindness. Br J Neurosurg. 2011;25:644-6.

5. Harrigan MR, Deveikis JP. Handbook of cerebrovascular disease and neurointerventional technique. Heidelberg: Springer; 2013.

6. Date I, Asari S, Ohmoto T. Cerebral aneurysms causing visual symptoms: their features and surgical outcome. Clin Neurol Neurosurg. 1998 100:259-67.

7. Jacobson DM. Symptomatic compression of the optic nerve by the carotid artery. Ophthalmology. 1999;106:1994-2004.
8. Sanders-Taylor C, Kurbanov A, Cebula H, Leach JL, Zuccarello M, Keller JT. The carotid siphon: a historic radiographic sign, not an anatomic classification. World Neurosurg. 2014;82:423-7.

9. Sano $\mathrm{H}$, Kato $\mathrm{Y}, \mathrm{Kiya} \mathrm{N}$, Ohkuma I, Ninomiya T, Kanno T. Classification and treatment of aneurysms of the internal carotid siphon. J Clin Neurosci. 1996;3:247-51.

10. Juvela S, Porras M, Poussa K. Natural history of unruptured intracranial aneurysms: probability of and risk factors for aneurysm rupture. J Neurosurg. 2000;93:379-87.

11. International Study of Unruptured Intracranial Aneurysms Investigators. Unruptured intracranial aneurysms - risk of rupture and risks of surgical intervention. N Engl J Med. 1998;339:1725-33.

12. Day AL. Aneurysms of the ophthalmic segment. J Neurosurg. 1990;72:677-91.

13. Vega-Basulto, S.D. et al. Aneurismas de la región de la arteria oftálmica. Neurocirugía [online]. 2006, vol.17, n.4, pp.303-316. Disponible en: http:// scielo.isciii.es/scielo.php?script=sci arttext\&pi$\mathrm{d}=\mathrm{S} 1130-14732006000400001$ \&lng=es\&nrm=iso

14. Silva Neto ÂR, Câmara RLB, Valença MM. Carotid siphon geometry and variants of the circle of Willis in the origin of carotid aneurysms. Arq Neuropsiquiatr. 2012;70:917-21.

15. Jason J. S. Barton MB. Automated Perimetry. En: Field of vision. First Edition. Daniel Tarsy. New Jersey: Humana Press; 2003.

16. Nieto MSG. Segmento clinoideo (C5) y espacio clinoideo. Revisión anatómica y su relevancia en cirugía neurovascular y de base de cráneo. Neuroeje. 2012;25:62-7. 